\title{
Note on magnitude estimation on digitized astronomical photographic plates
}

\author{
R. Hudec ${ }^{1,3}$ and E. Splittgerber ${ }^{2}$ \\ ${ }^{1}$ Czech Technical University in Prague, Faculty of Electrical Enineering, \\ Prague, Czech Republic, (E-mail: hudecren@fel.cvut.cz) \\ 2 Sonneberg Observatory, Sonneberg, Germany \\ ${ }^{3}$ Kazan Federal University, Kazan, Russian Federation
}

Received: June 10, 2020; Accepted: June 30, 2020

\begin{abstract}
The scanned astronomical archival plates offer the possibility of alternative analyses on these data. Apart from a complete evaluation by sophisticated computer programs, the recent efforts offer an alternative fast method of eye estimation method using scanned plates exhibited on computer screens. This alternative method has been investigated by analyzing the light curve of XX Cep on several hundred scanned sky patrol plates from the Sonneberg Observatory plate archive.

Key words: sky surveys - astronomical photography - astronomical plate digitization
\end{abstract}

\section{Introduction}

There are about 10 million astronomical photographic glass plates and negatives in the plate archives over the globe (Hudec, 2019, 2014, 2007). A large fraction of them were taken for purposes of variable star research. The methods applied in the past to approach and analyze these data included plate photometry by dedicated plate photometers (e.g. iris photometers) as well as eye estimate methods, and more recently the computer analyses based on scanned data. The method of eye estimates, based on the original Argelander method or similar alternative methods, was widely used over the past 100 years in variable star research and has resulted in numerous scientific publications until recently, e.g. (Hudec et al., 2013). Over many decades, it was the mostly used method to access the data recorded in astronomical archival plates and to yield the variable star magnitudes. At many centers of variable star research, such as the Sonneberg Observatory in Germany (Hudec, 2007; Kroll, 2009), it represented the mostly used method (Hudec, 1999). The obvious preference of this method was that it was fast (in contrast to plate photometers which were slow), on the other side it has required some non-trivial skills and hence only experienced observers were able to achieve a reasonable accuracy of the measurements. However, an experienced observer was able to provide accuracy of measurements analogous 
to or even in some cases exceeding those obtained with plate photometers, see e.g.(Hudec \& Wenzel, 1976).

Recently, many observatories with plate collections have started (partly extended) scanning efforts, both with dedicated custom-made plate scanners as well as with commercial flatbed scanners. One of the most extended scanning was at the Sonneberg Observatory, Germany, where the largest European astronomical plate archive with about 280000 plates is deposited. So far 237430 plates have been scanned there. The data were stored on DVDs and hard discs (Kroll, 2009) ${ }^{1}$.

In addition to that, there are two major scanning projects with automated pipelines to access and evaluate digitized plate data:

(i) APPLAUSE (Germany Hamburg, Bamberg, Potsdam) 70276 plates so far $^{2}$, (Tuvikene et al., 2014) and (ii) DASCH Harvard College Observatory (HCO) USA 395921 plates so far (Grindlay et al., 2012) ${ }^{3}$.

As a consequence of converting the information recorded on astronomical sky plates, novel methods of data mining and data analyses have appeared (Hudec, 2019; Hudec \& Hudec, 2014). While the most important one rely on evaluation of the digitized data by powerful computers and dedicated software programs, there is another interesting alternative as described in this contribution, and this is the eye estimate on a computer screen instead of with a microscope and original plates.

The obvious preference of this method is that it is fast (as in the case of estimates on original plates) but the additional preference is that this work can be done remotely also without approaching the plates.

\section{XX Cep as a test object}

As it was not very clear what the accuracy in this case will be, we have performed a test study. We have selected a variable eclipsing binary XX Cep as the test object. XX Cephei (HD 222217) was discovered photographically by Schneller (1928). Interest in this partially eclipsing Algol system centered on the systems possible apsidal motion. The evidence for apsidal motion was based on the eccentric spectroscopic orbit determined by Struve (1946), a displaced secondary minimum reported by Iljasova (1946), Fresa (1956), and Lavrov (1957), and possible cyclic changes in the period, see Mayer (1984). Mardirossian et al. (1980) obtained a photometric solution from a reanalysis of the Fresas data. The most recent photometric solution was by Bonifazi \& Guarnieri (1986) based on observations made with the $60 \mathrm{~cm}$ telescope at Bologna Observatory.

\footnotetext{
${ }^{1}$ https://www.plate-archive.org/applause/wp-content/uploads/2019/04/

Kroll_PhotographicAndDigitalSkySurveysAtSonnebergObservatory.pdf

${ }^{2}$ https://www.plate-archive.org/applause/

${ }^{3}$ http://dasch.rc.fas.harvard.edu/lightcurve.php
} 
$\mathrm{XX}$ Cephei is a system with a changing period, probable mass exchange, and a possible light-time effect from a third stellar companion. It was shown that a continuous period change coupled with a light-time effect is a plausible explanation for the period changes (Angione \& Sievers, 2006). A spectroscopic search for evidence of mass transfer and features of the cooler component yielded no positive results (Angione \& Sievers, 2006).

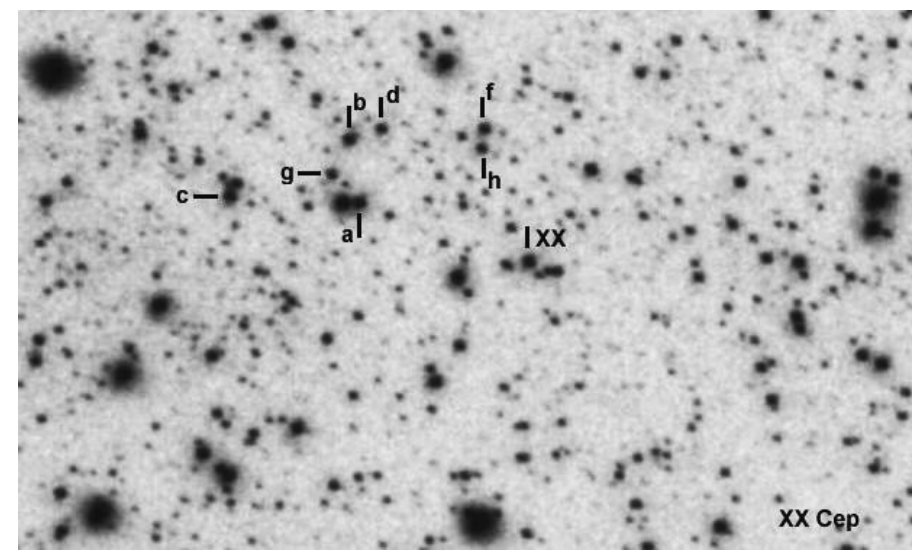

Figure 1. The area of XX Cep on the scanned Sonneberg sky patrol plate with the variable star indicated, as well as comparison stars used for the measurements.

The star was measured by one of us (ES) on 950 blue sensitive sky patrol plates taken between 1958 and 1995 by the patrol cameras at the Sonneberg Observatory. For the study, the original scanned data were unzipped and then transferred from a tiff format to a jpeg image format with following visualization on a laptop screen (Fig. 1). Then the eye estimation method was applied in a similar manner as usual. We note that the star was about 4 magnitudes brighter than the limiting magnitude on the negatives used. Typically, stars of this magnitude have the best accuracy in case of eye estimates. The resulting orbital light curve of the test variable star is shown in Fig. 2. The elements from the literature (Bonifazi \& Guarnieri, 1986) have been used to construct this curve. It is obvious that the mean measurement error in this case amounts to about $0.1 \mathrm{mag}$, which is a typical value for an eye estimation method of this type of plate material.

\section{Discussion}

The amateur astronomers have always provided a valuable contribution to data analyses recorded on astronomical archival plates. They were regular visitors to many observatories with large plate collections. Nowadays, after the relevant 


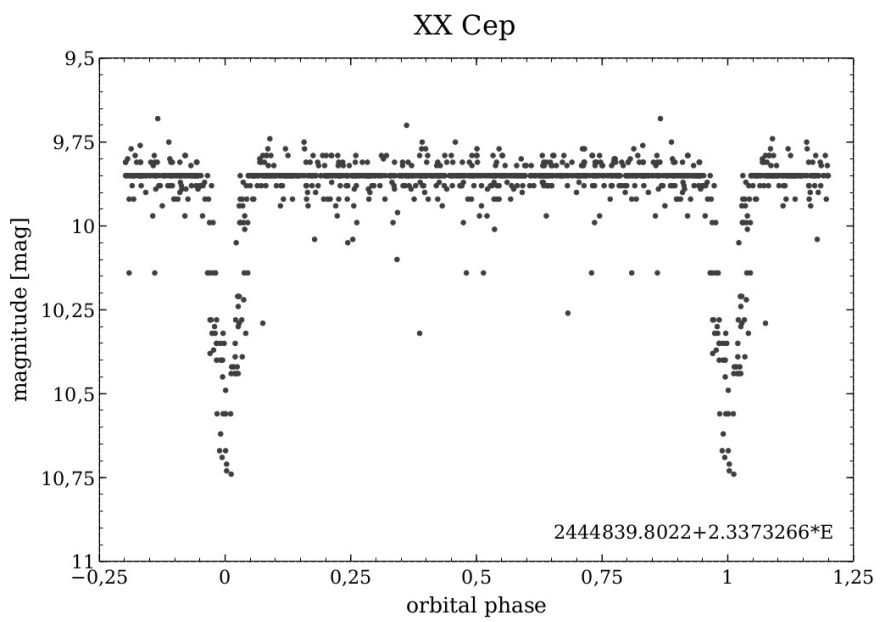

Figure 2. The orbital light curve of XX Cep generated by the method described and discussed in this paper.

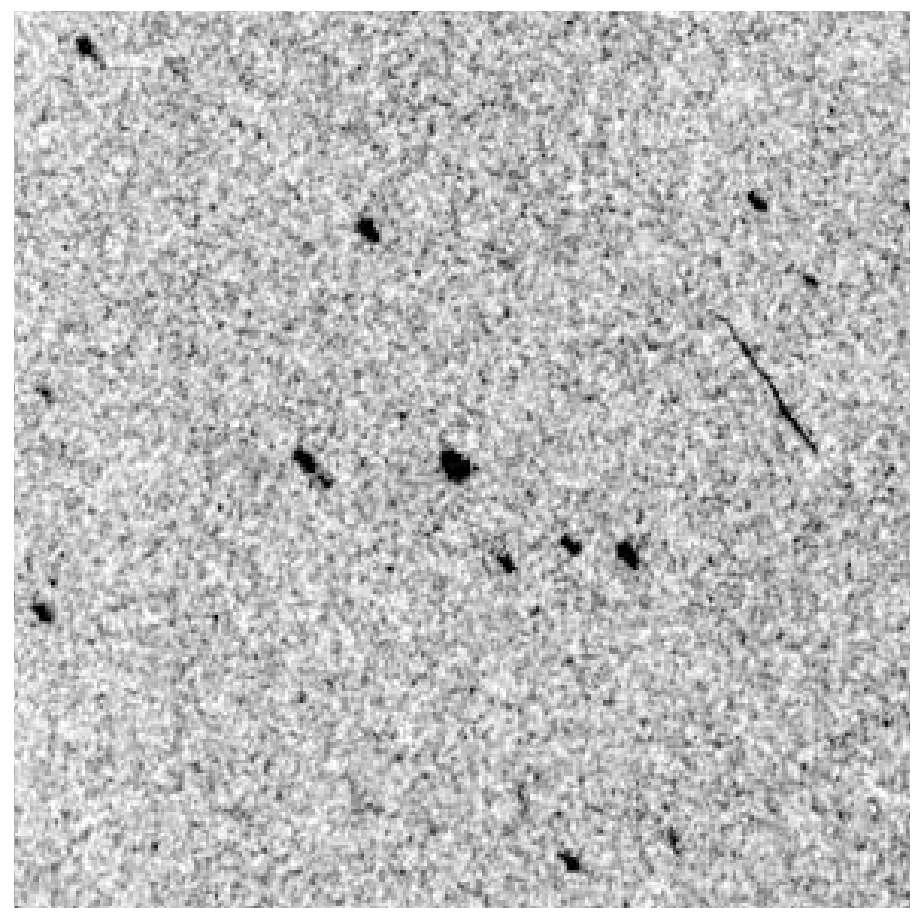

Figure 3. The area of XX Cep on the scanned HCO plate, the target is in the center. 


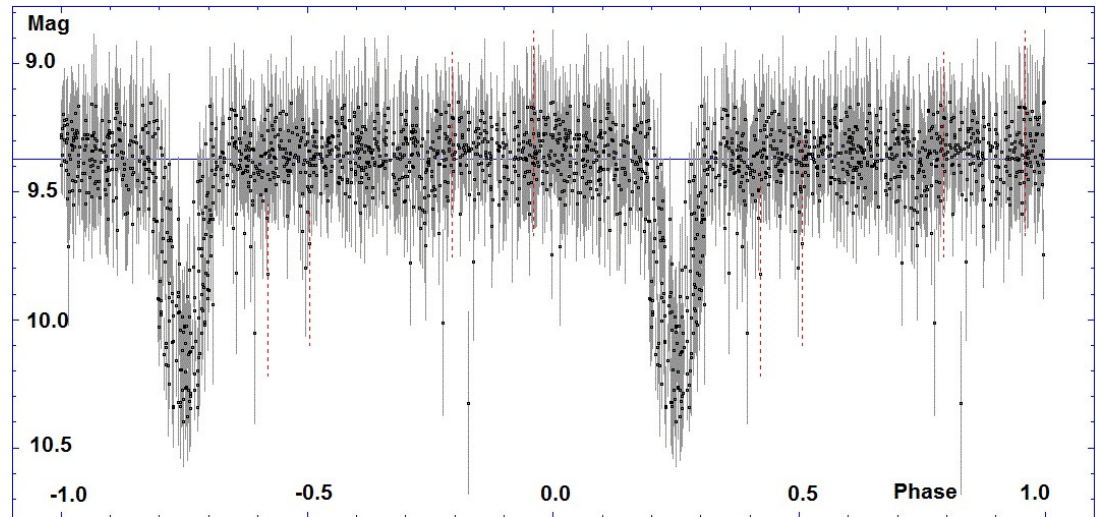

Figure 4. The orbital light curve of XX Cep generated by the automated DASH pipeline.

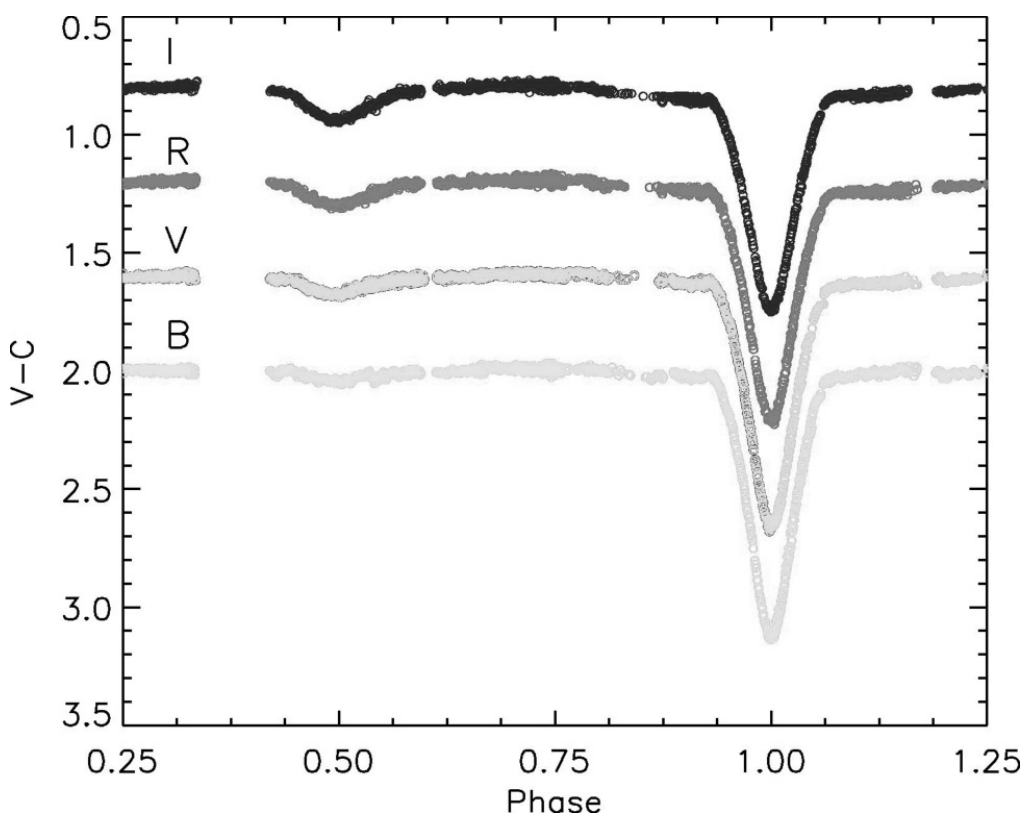

Figure 5. The orbital light curve of XX Cep based on CCD observations by $40 \mathrm{~cm}$ and $35 \mathrm{~cm}$ telescopes (for comparison) (Hosseinzadeh et al., 2014). It shows that the small secondary minimum around phase 0.5 is almost invisible in the B filter (note that the Sonneberg photographic data used in our study are blue senstitive, i.e. close to it). 
plates were fully scanned, they have an alternative possibility, namely to analyze the digitized plates remotely on their laptops. While the emphasis of plate analyses must rely on a sophisticated evaluation of scanned data by computers and dedicated software packages, at the same times the eye estimates can contribute to some special related aspects.

For a comparison, we have created an analogous light curve of XX Cep using the automated DASCH pipeline analyzing scanned HCO plates. (Figs. 3 and 4). It is evident that the scatter in the generated light curve exceeds the scatter of the light curve obtained by our method described in this paper. The small secondary minimum in the orbital light curve of XX Cep visible in data sets provided by photoelectric photometry and CCD telescopes is invisible in our orbital light curve (Fig. 2) but we note that, albeit the photographic observations are always limited in their accuracy covering small details less than $0.1 \mathrm{mag}$, the amplitude of secondary minima depends on wavelength, being less prominent in the blue band (Fig. 5).

\section{Conclusions}

We conclude that the accuracy of the eye estimation method performed with digitized plate data visualized on a computer (laptop) screen is similar to that obtained for direct eye estimates on original plates with the use of a microscope.

Acknowledgements. The authors are extremely thankful to the staff of the Sonneberg Observatory. This work was partly supported by GACR grant 13-33324S and by the MSMT Mobility project 8J18AT036. We thank the referee, Dr. Sergei Shugarov, for his valuable comments improving the paper.

\section{References}

Angione, R. J. \& Sievers, J. R., The Algol System XX Cephei. 2006, Astron. J., 131, 2209, DOI: $10.1086 / 501009$

Bonifazi, A. \& Guarnieri, A., Photoelectric study of the low-mass semidetached system XX Cephei. 1986, Astron. Astrophys., 156, 38

Fresa, A., Curva di luce fotoelettrica ed orbita della variabile ad eclisse X X Cephei. 1956, Memorie della Societ Astronomia Italiana, 27, 299

Grindlay, J., Tang, S., Los, E., \& Servillat, M., Opening the 100-Year Window for Time-Domain Astronomy. 2012, in IAU Symposium, Vol. 285, New Horizons in Time Domain Astronomy, ed. E. Griffin, R. Hanisch, \& R. Seaman, 29-34

Hosseinzadeh, B., Pazhouhesh, R., \& Yakut, K., Observations, light curves analysis and pulsation behavior of the Algol-type eclipsing binary system XX Cep. 2014, New Astronomy, 27, 95, DOI: 10.1016/j.newast.2013.08.005

Hudec, R., An introduction to the world's large plate archives. 1999, Acta Historica Astronomiae, 6, 28 
Hudec, R. 2007, Astrophysics with Astronomical Plate Archives, ed. A. P. Lobanov, J. A. Zensus, C. Cesarsky, \& P. J. Diamond, 79

Hudec, R., Digitized Astronomical Photographic Archives as Large Area Sky Survey of Large Amount of Astronomical Data. 2014, in Proceedings of Frontier Research in Astrophysics (FRAPWS2014) held 26-31 May, 2014 in Mondello (Palermo), Italy. Online at https://pos.sissa.it/237/039/pdf, 39

Hudec, R., Astronomical photographic data archives: Recent status. 2019, Astronomische Nachrichten, 340, 690, DOI: 10.1002/asna.201913676

Hudec, R., Bašta, M., Pihajoki, P., \& Valtonen, M., The historical 1900 and 1913 outbursts of the binary blazar candidate OJ287. 2013, Astron. Astrophys., 559, A20, DOI: 10.1051/0004-6361/201219323

Hudec, R. \& Hudec, L., US Astronomical Photographic Data Archives: Hidden Treasures and Importance for High-Energy Astrophysics. 2014, in Multifrequency Behaviour of High Energy Cosmic Sources, 316-319

Hudec, R. \& Wenzel, W., Observations of HZ Her/Her X-1 on Sonneberg Astrograph Plates. 1976, Bulletin of the Astronomical Institutes of Czechoslovakia, 27, 325

Iljasova, N., XX Cephei. 1946, Engelhardt Astron. Obs. Bull., 24, 12

Kroll, P., Real and Virtual Heritage - The Plate Archive of Sonneberg Observatory - Digitisation, Preservation and Scientific Programme. 2009, in Cultural Heritage of Astronomical Observatories: From Classical Astronomy to Modern Astrophysics, ed. G. Wolfschmidt, 311-315

Lavrov, M. I., The Eclipsing Variable Star XX Cephei. 1957, Peremennye Zvezdy, 12, 21

Mardirossian, F., Mezzetti, M., Cester, B., Giuricin, G., \& Russo, G., Revised photometric elements of five possible sd-d systems. 1980, Astron. Astrophys., Suppl., 39, 235

Mayer, P., Periodic Terms in the Light Elements of XX CEP and RW Per. 1984, Bulletin of the Astronomical Institutes of Czechoslovakia, 35, 180

Schneller, H., Das System RS Canum venaticorum. 1928, Astronomische Nachrichten, 233, 361, DOI: 10.1002/asna.19282332202

Struve, O., Spectrographic Observations of Eleven Eclipsing Binaries. 1946, Astrophys. J., 103, 76, DOI: 10.1086/144790

Tuvikene, T., Edelmann, H., Groote, D., \& Enke, H., Work flow for plate digitization, data extraction and publication. 2014, in Astroplate 2014, 127 